\title{
Application of spin markers for study of liposome prepared by the modified reverse-phase evaporation method
}

\author{
Danuta Pentak $^{\text {a }}$, Wiesław W. Sułkowski a,*, Agnieszka Wolińska ${ }^{\text {a }}$, Sławomir Maślanka a \\ Barbara Bojko $^{\text {b, }}$, Małgorzata Maciążek ${ }^{\mathrm{b}}$, Joanna Równicka ${ }^{\mathrm{b}}$ and Anna Sułkowska ${ }^{\mathrm{b}}$ \\ ${ }^{a}$ Department of Environmental Chemistry and Technology, Institute of Chemistry, University of Silesia, \\ 40-006 Katowice, Poland \\ ${ }^{\mathrm{b}}$ Department of Physical Pharmacy, Medical University of Silesia, 41-200 Sosnowiec, Poland
}

\begin{abstract}
The EPR spectroscopy was used to determine the structure and physicochemical properties of liposomes prepared from L- $\alpha$-phosphatidylcholine dipalmitoyl (DPPC) by the modified reverse-phase evaporation method (mREV). EPR study was carried out in the temperature range from $297 \mathrm{~K}$ to $340 \mathrm{~K}$ i.e. below and above the phase transition temperature $T_{\mathrm{C}}$ of DPPC. On the basis of EPR spectra of spin marker 2,2,6,6-tetramethyl-1-piperidinyloxy (TEMPO) incorporated into the liposome, the parameter $f$ was determined. TEMPO dissolves easily in water and in the fluid lamellar smectic liquid-crystaline phase of lipid bilayer but is largely excluded from the solid, gel-phase. Thus TEMPO can be used to observe the change in the partition between aqueous and fluid lipid regions. The change in the relative value of $f$ as a function of temperature for DPPC shows that, in the presence of water excess, this phospholipids undergoes a transition from a "gel phase" to a lamellar smectic liquid crystalline phase. On the basis of EPR spectra of spin marker 2-(3carboxypropyl)-4,4-dimethyl-2-tridecyl-3-oxazolidinloxyl (5-DOXYL) incorporated into liposome, the parameter $a_{\mathrm{N}}^{\prime}$ was determined. The isotropic ${ }^{14} \mathrm{~N}$-hyperfine coupling constant $a_{\mathrm{N}}^{\prime}$ of nitroxide spin label depends on the local environmental polarity. The increase of $a_{\mathrm{N}}^{\prime}$ value reflects the rise of polarity of spin label environment. Temperature, cholesterol and $\mathrm{pH}$ dependent structural changes were also described.
\end{abstract}

Keywords: Liposomes, mREV, 5-DOXYL, TEMPO

\section{Introduction}

Liposomes are spherical vesicles integrated by one or more phospholipid bilayers that encapsulate a part of the aqueous media in which they are suspended. The formation of liposomes occurs when phospholipids are dispersed in the water as a result of unfavourable interactions between phospholipids and water. The amphiphilic character of phospholipids and their capability of forming closed structures allow for both hydrophobic and hydrophilic molecules to be trapped inside liposomes.

Liposomes can be classified into three groups: multilamellar vesicles (MLVs), small unilamellar vesicles (SUVs) and large unilamellar vesicles (LUVs). MLVs are generally heterogeneous in nature and may have several compartments. They are easy to prepare and can vary in size from 0.5 to $5 \mu \mathrm{m}$. SUVs range in size from 20 to $50 \mathrm{~nm}$, have a spherical shape, and are homogeneous in nature. Finally, LUVs are larger (200-1000 nm), giving them greater space for encapsulation the of aqueous medium.

\footnotetext{
*Corresponding author: W.W. Sułkowski, Department of Environmental Chemistry and Technology, Institute of Chemistry, University of Silesia, Szkolna 9, 40-006 Katowice, Poland. Tel./Fax: +48 32 3591371; E-mail: wsulkows@ uranos.cto.us.edu.pl.
} 
Liposome has been used as drug delivery vehicle or biomembrane model due to the advantages such as ease and convenience of preparation, biocompatibility, low toxicity and biodegradability. This selfassembled structures are interesting not only as drug delivery systems but also for biochemistry and molecular biology, cosmetics and food technology [1].

The structure and dynamics of membrane lipids and their relation to the membrane function are important problems in membrane biophysics. Electron paramagnetic resonance (EPR) is extremely useful in the studies of membrane dynamics and microenvironment of the reaction field such as microviscosity or micropolarity.

Membrane studies by EPR are based on the use of a versatile nitroxide probe. Nitroxides are stable free radicals having EPR spectra sensitive to the molecular microenvironment. Several reviews have described the theory of nitroxide EPR spectral analysis and applications of nitroxides for membrane studies [2].

Studies on preparation of liposomes of different dimensions and number of layers are conducted from over 30 years [3]. The methods of investigation of the liposome properties were also worked out [4].

However the problem how to obtain the liposomes of universal application remains insoluble. Therefore the modification of the methods of liposome preparation is still conducted. The description of the physicochemical properties of liposomes obtained with these modified methods is needed in order to estimate the usefulness of the applied methodology and to define the field of the potential application of the obtained liposomes [3,4].

In the present work we characterize the polarity profiles derived from 5-DOXYL spin-label measurements with DPPC and DPPC/Chol membranes using an analytical expression that is sigmoidal and decays exponentially toward the mid-plane of the membrane. This profile is thermodynamically based and may be transformed directly into the membrane permeability barrier for polar solutes.

On the basis of TEMPO spin-label studies we showed how the paramagnetic resonance spectra can be used to determine parameter $f$ which is approximately equal to the fraction of spin label dissolved in the fluid lipid phase of the sample at a given temperature. The change in the relative values of $f$ as a function of temperature for DPPC is due to the fact that, in the presence of excess water, this phospholipid undergoes a transition from a "gel phase" to a lamellar smectic liquid crystalline phase.

\section{Materials and methods}

\subsection{Materials}

L- $\alpha$-phosphatidylcholine dipalmitoyl (1,2-dihexadecanoyl-sn-glycerol-3-phosphocholine) $99 \%$ (DPPC), the spin markers 2-(3carboxypropyl)-4,4-dimethyl-2-tridecyl-3-oxazolidinyloxyl free radical (5-DOXYL) and 2,2,6,6-tetramethyl-1-piperidinyloxy (TEMPO) were purchased from Sigma-Aldrich Chem. Co., $\alpha, \alpha, \alpha$-Tris-(hydroxymethyl)-methylamin $99.9 \%$ (TRIS) was purchased from Fluka, cholesterol (5-cholesten-3 $\beta$-ol) 99+\%, chloroform, dichloromethane and hydrochloric acid were purchased from POCH, Gliwice, Poland.

\subsection{Liposome preparation and characteristics}

We obtained small liposomes (DPPC/Chol) by the modified reverse-phase evaporation method (mREV) [5-8] using the DPPC:Chol molar ratio: 4.00:1.00, 4.00:0.50 and 4.00:0.25. Tris-HCl buffer (pH 1.9, 5.0, 7.4, 8.0 or 8.4) was applied. For EPR study $0.016 \mathrm{ml}$ of the spin marker (5-DOXYL) 
(12.5 $\mathrm{mg} / \mathrm{ml}$ in chloroform) and $0.096 \mathrm{ml}$ of the spin marker (TEMPO) $(0.78 \mathrm{mg} / \mathrm{ml}$ in chloroform) were added to the preparation mixture. The liposomes not containing cholesterol were also prepared. The preparation was carried out at $318 \mathrm{~K}$. The average time of liposome preparation did not exceed 12 minutes. The excess of spin marker was separated by treble centrifugation using High Speed Brushless Centrifuge MPW - 350, with the speed of 4500 revs. per minute, for a period of $10 \mathrm{~min}$. Liposomes obtained by mREV method were extruded through a filter of $100 \mathrm{~nm}$ in diameter using LiposoFast Basic apparatus produced by Avestin [9].

\subsection{Electron paramagnetic resonance}

Electron paramagnetic resonance (EPR) technique was used to monitor the molecular dynamics of lipids. This spectroscopic technique allowed us to detect changes in the spin tropic movement of an unpaired electron. Biomolecules, as DPPC or cholesterol, which do not contain unpaired electrons, can be studied by EPR when they are surrounded or chemically bonded to a stable free radical. 5-DOXYL (Fig. 1) and TEMPO (Fig. 2) were used as a spin marker.

The EPR measurements were carried out on a Bruker EMX spectrometer at the X-band ( $9 \mathrm{GHz})$, equipped with Bruker $\mathrm{N}_{2}$ temperature controller in the temperature range 297-340 K, and the constant temperature $\pm 0.5 \mathrm{~K}$ was maintained during the experiment. All measurements were performed in a triplicate. The spectra were recorded on microwave power $20.070 \mathrm{~mW}$, with $4.48 \times 10^{4}$ signal amplification, $0.80 \mathrm{G}$ modulation amplitude, sweep time $20.973 \mathrm{~s}$ and number of scans -10 . For the EPR experiment $0.1 \mathrm{ml}$ of liposome sample solutions were kept in closed quartz capillaries.<smiles>CCCCCCCCCCCCCC1(CCCC(=O)O)OCC(C)(C)N1O</smiles>

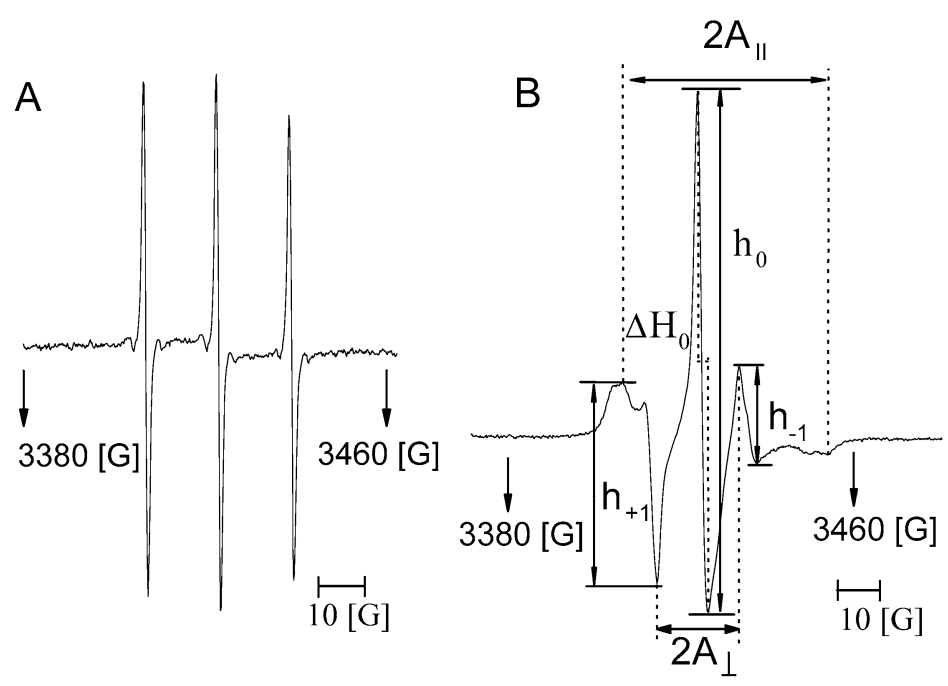

Fig. 1. Structural formula and typical EPR spectra of spin marker 5-DOXYL at $300 \mathrm{~K}$ : (A) in solution, (B) after incorporation into the liposome membrane. 

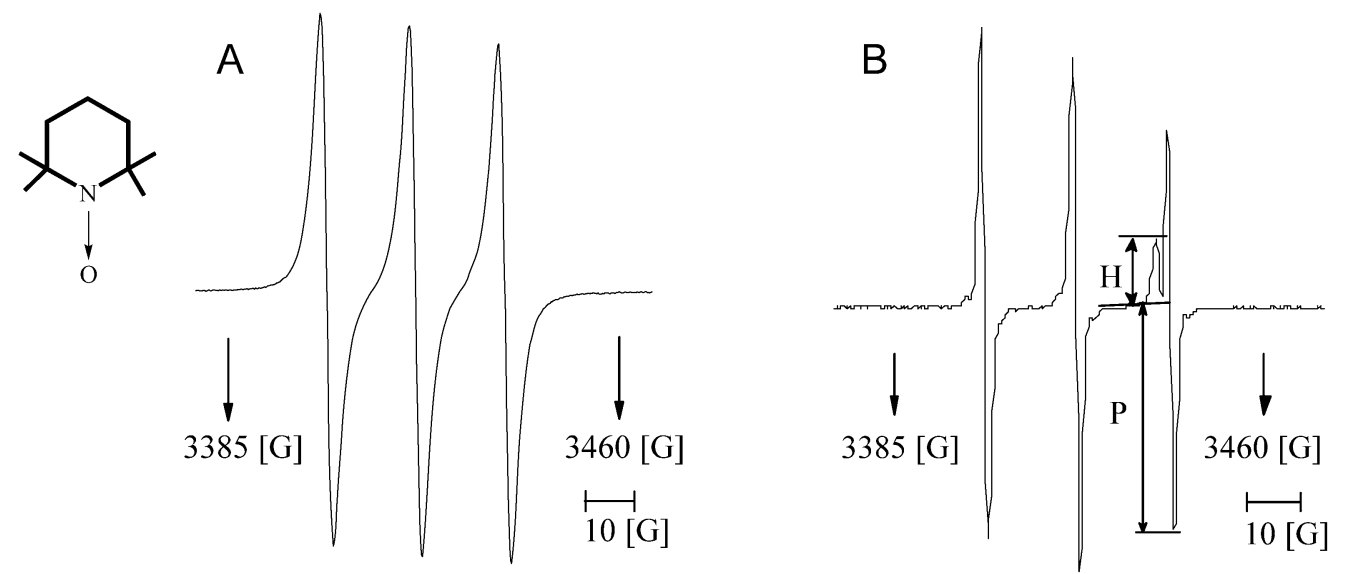

Fig. 2. Structural formula and typical EPR spectra of spin marker TEMPO at $335 \mathrm{~K}$ : (A) in solution, (B) after incorporation into the liposomes.

Isotropic ${ }^{14} \mathrm{~N}$-hyperfine constants were determined from relation (1):

$$
a_{\mathrm{N}}^{\prime}=\frac{1}{3}\left(A_{\mathrm{II}}+2 A_{\perp}\right)
$$

where $A_{\perp}$ and $A_{\mathrm{II}}$ are the hyperfine constants determined with the magnetic field perpendicular and parallel to the membrane normal. The latter were determined from the outer and inner spectral hyperfine splittings (Fig. 1B). For spectra of spin labels close to the terminal methyl group of the lipid chains, the spectral anisotropy is small and not well resolved.

\section{Results and discussion}

\subsection{EPR study - polarity profiles from spin labels}

The isotropic ${ }^{14} \mathrm{~N}$-hyperfine coupling constant $a_{\mathrm{N}}^{\prime}$, of nitroxide spin labels is dependent on local environmental polarity. Therefore the dependence of $a_{\mathrm{N}}^{\prime}$ in fluid phospholipid bilayer membranes on the C-atom positions, $n$, of the nitroxide in the $s n-2$ chain of a spin-labeled diacyl glycerophospholipid can be used to estimate the trans-membrane polarity profile [10].

Isotropic ${ }^{14} \mathrm{~N}$-hyperfine constants were determined on the basis of relation (1) from the outer and inner spectral hyperfine splittings (Fig. 1B). The change of the polarity of the phospholipid membranes with and without cholesterol is shown in Fig. 3.

The increase of $a_{\mathrm{N}}^{\prime}$ parameter value reflects the increase of membrane polarity [10]. The sigmoidal curve, characterizing the changes of the parameter $a_{\mathrm{N}}^{\prime}$ values, also shows changes of fluidity of phospholipid membranes [10]. For all studied liposomes $a_{\mathrm{N}}^{\prime}$ parameter decreases with the rise of temperature (Fig. 3). Therefore one can assume that the membrane fluidity also decreases. The value of $a_{\mathrm{N}}^{\prime}$ parameters diminishes when $\mathrm{pH}$ increases from 1.9 to 8.4 at temperature range $297-310 \mathrm{~K}$ (Fig. 4). Above the temperature of phase transitions $T_{\mathrm{C}}(314 \mathrm{~K})$ the inversion of the $a_{\mathrm{N}}^{\prime}$ value with increase of temperature can be seen. This effect is observed for liposomes prepared both in acidic and basic medium (Fig. 4). The inversion of $a_{\mathrm{N}}^{\prime}$ parameter value can be explained by the perpendicular displacement of the spin marker 5-DOXYL to the membrane surface, along the aliphatic chains of phospholipids. 


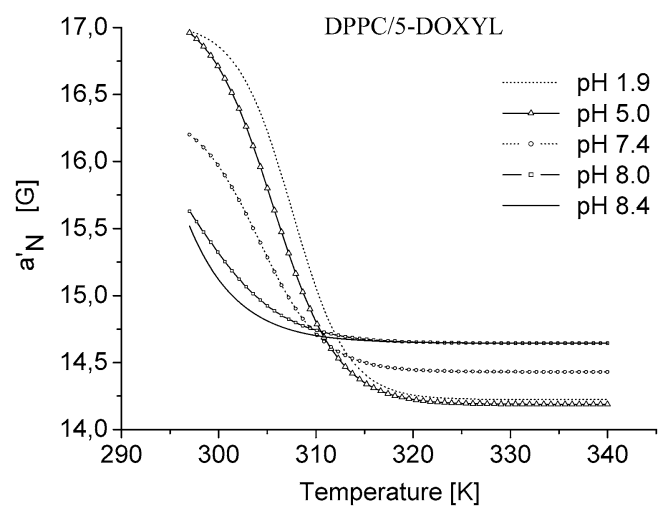

(a)

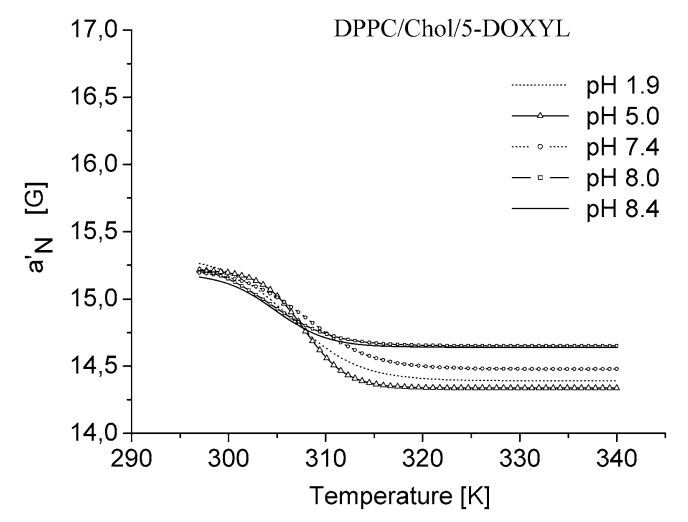

(b)

Fig. 3. Effect of $\mathrm{pH}$ temperature and cholesterol on polarity of the studied liposomal membranes. Liposomes were obtained from: (a) DPPC/5-DOXYL, (b) DPPC/Chol/5-DOXYL (DPPC:cholesterol molar ratio 4.00:1.00).

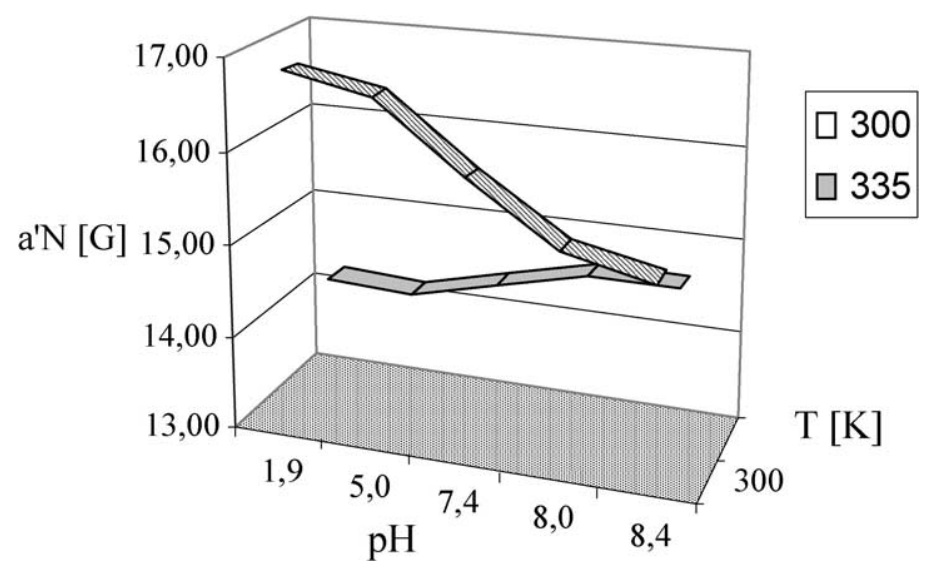

Fig. 4. Effect of $\mathrm{pH}$ and temperature on the motion of spin marker 5-DOXYL along the perpendicular to the surface of membrane. Liposomes were formed from DPPC; spectra were recorded below $T_{\mathrm{C}}(300 \mathrm{~K})$ and above $T_{\mathrm{C}}(335 \mathrm{~K})$.

Such a movement can be induced by both change of $\mathrm{pH}$ and rise of temperature. The results confirm the increase of the polarity of liposomal membranes formed from DPPC with increase of the distance of the spin marker from the membrane surface. A similar effect has been described previously [10]. The change of $\mathrm{pH}$ of the buffer, in which liposomes are prepared, from the acidic to the basic one, induces a change of the direction of the spin marker movement. In a basic environment, above the temperature of phospholipid phase transitions the spin marker probably displaces a deflection of the phospholipid polar "heads" into the bilayer (Fig. 5). Then the polarity of liposome decreases. In an acidic environment, above the temperature of phase transition such a change of deflection of phospholipid polar "heads" was observed that it showed the displacement of the spin marker movement towards the membrane surface.

The change of ionization of phosphate groups of phosphatidylcholine can facilitate the displacement of the spin marker along the normal to the surface of the phospholipid membrane. It can be related to the decline or appearance of electrostatic and/or hydrogen bondings formed by phosphate groups. These interactions can cause conformational changes of phosphate groups in the way presented in Fig. 5. 


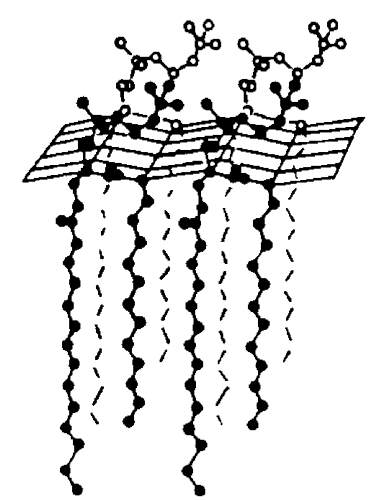

(a)

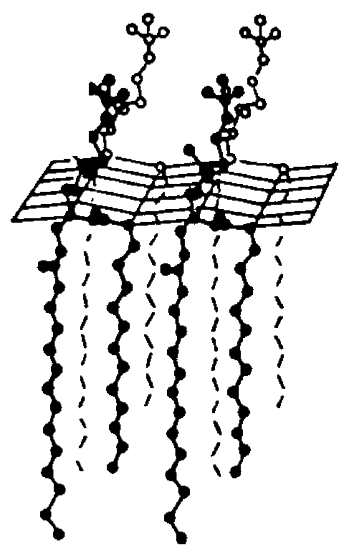

(b)

Fig. 5. Schematic drawing shows the conformation of polarity phospholipids head group; (a) conformation with parallel position of phospholipid "head" to the membrane surface, (b) conformation with perpendicular position of polar group to the membrane surface.

\subsection{EPR study - partitioning of TEMPO between the hydrocarbon and aqueous phases}

The EPR study of liposomes with the spin marker TEMPO has been carried out in order to estimate the usefulness of the obtained liposomes to transport hydrophilic drugs. This spin marker was chosen due to the similarity of its properties to those of hydrophilic drugs. The EPR study of liposomes labeled by the spin marker TEMPO allows us to determine the content of the marker in both the hydrophobic $(H)$ and hydrophilic $(P)$ parts of the liposomal membrane basing on the parameter $f(2)$ estimation (Fig. 6).

$$
f=H /(H+P) .
$$

The EPR spectrum of spin marker TEMPO incorporated into liposomes comprises two superposed spectra, i.e. spectrum of the marker located in the hydrophobic part of the membrane and spectrum of the marker located in the hydrophilic one. Studies were carried out for liposomes formed with DPPC/TEMPO, DPPC/Chol/TEMPO in Tris- $\mathrm{HCl}$ buffer with $\mathrm{pH}$ 1.9, 5.0, 7.4, 8.0 and 8.4 respectively.

The content of the spin marker located in the hydrophilic part of the membrane $(H)$ increases when temperature rises from 305 to $340 \mathrm{~K}$ (Fig. 6). The changes in the EPR spectrum of the spin marker TEMPO can be explained by the increasing penetration of the spin marker between the hydrophilic and hydrophobic parts of the liposome. The increase of value $H$ causes the increase of parameter $f$ (Fig. 7).

This is probably connected with the loosening of the membrane and with penetration of water between the hydrocarbon chains. The results obtained are in accordance with literature data [11-14]. The presence of cholesterol in the liposomal membrane causes the decrease of the partition coefficient $f$ value. This is probably due to the stiffening affect of sterol and the inhibition of the extent of the spin marker penetration between the parts of the membrane. The partition coefficient $f$ changes with temperature (Fig. 7). These dependences allow us to determine the phase transition temperature $T_{\mathrm{C}}$ of phospholipid.

It was confirmed that $T_{\mathrm{C}}$ value depends not only on the phospholipid used for the liposome membrane preparation but also on the $\mathrm{pH}$ of the buffer used for its preparation and on the cholesterol content. All these parameters determine the thermotropic properties of phospholipids forming liposomes.

The addition of cholesterol induces the increase of $T_{\mathrm{C}}$ value. The highest values of phase transition temperature $T_{\mathrm{C}}$ were determined for liposomes obtained in buffer with $\mathrm{pH}$ equal 1.9 (Fig. 7). The values 

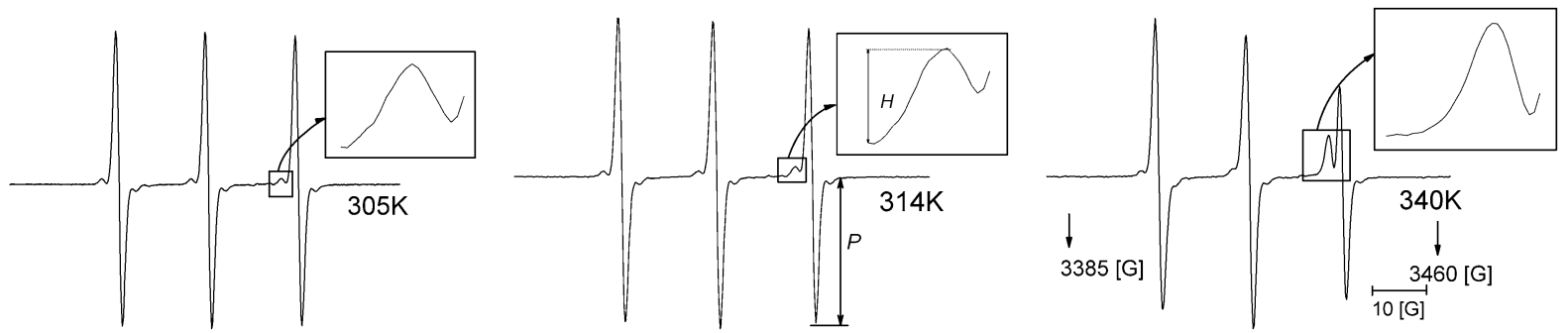

Fig. 6. Superposition of two spectra: spin label TEMPO located in the hydrophobic part of the membrane $(H)$ and spin label located in the hydrophilic part of the liposome $(P)$. Liposomes were obtained from DPPC/TEMPO Tris- $\mathrm{HCl} 1.9 \mathrm{pH}$; temperature $305 \mathrm{~K}, 314 \mathrm{~K}, 340 \mathrm{~K}$.
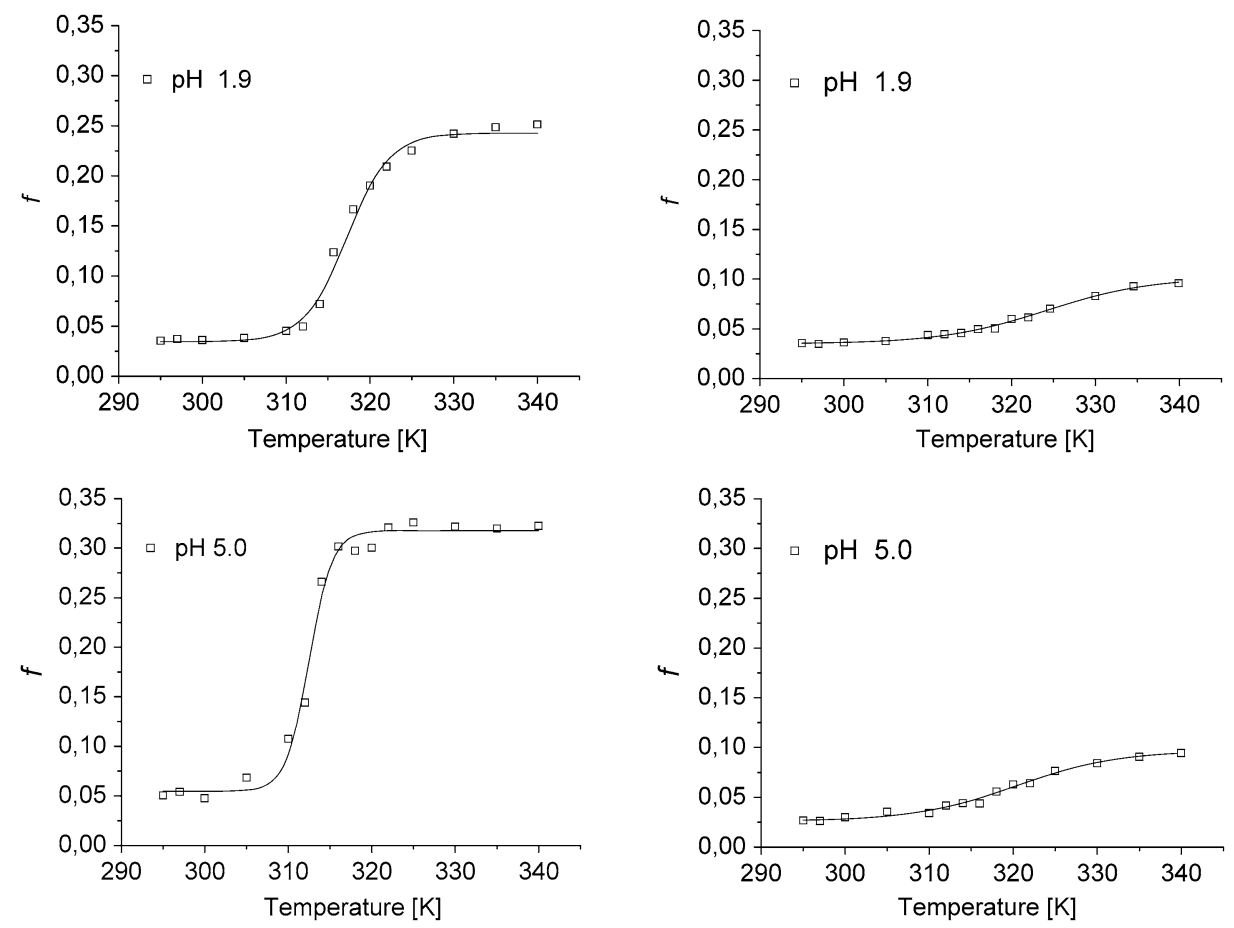

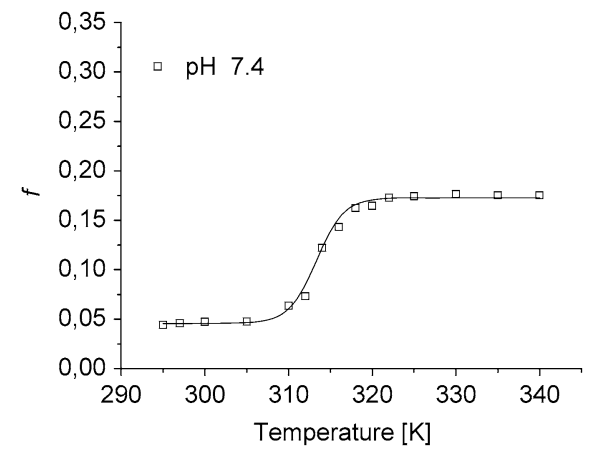

(a)

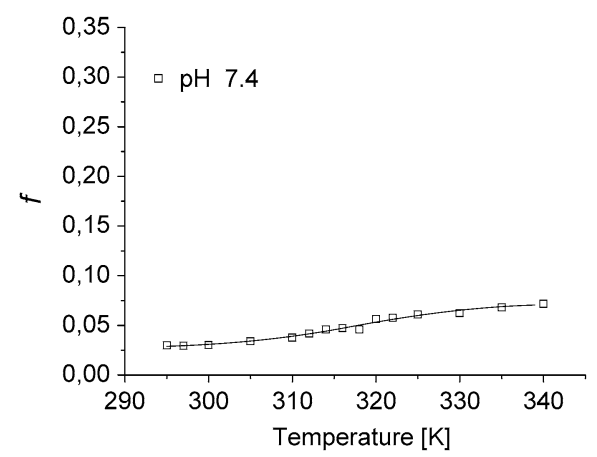

(b)

Fig. 7. Effect of temperature and cholesterol on the $f$ parameter values for liposomes formed from (a) DPPC/TEMPO, (b) DPPC/Chol/TEMPO (DPPC:cholesterol ratio 4.00:1.00). Temperature range 297-340 K, pH 1.9, 5.0, 7.4, 8.0, 8.4. 

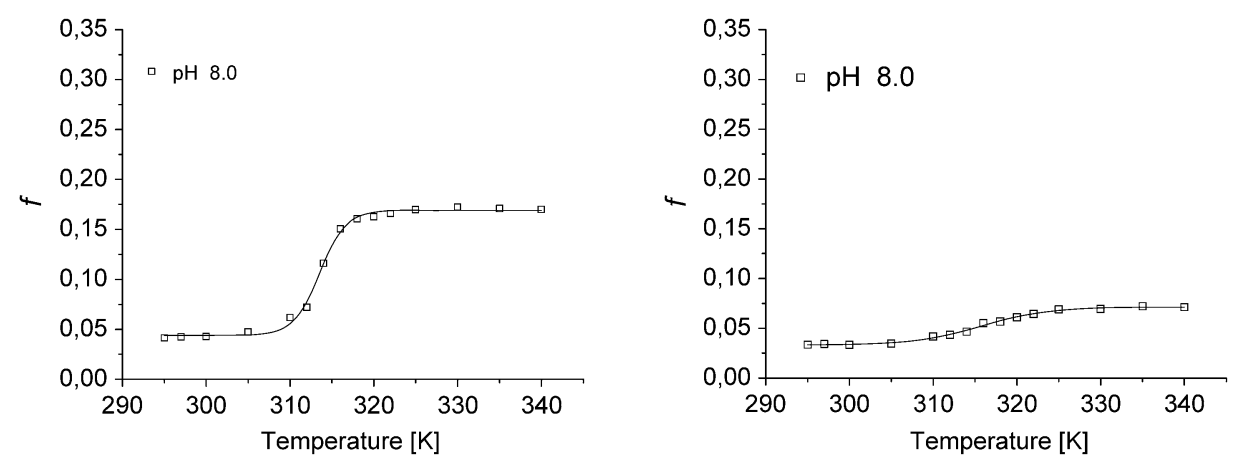

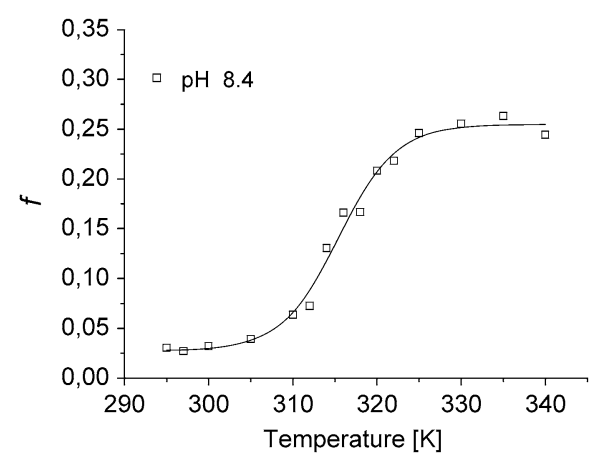

(a)

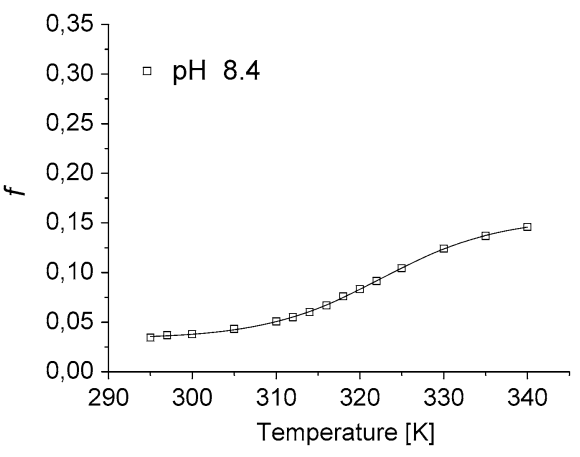

(b)

Fig. 7. (Continued).

Table 1

The effect of $\mathrm{pH}$ of the buffer on the temperature of phase transition $T_{\mathrm{C}}$

\begin{tabular}{ccc}
\hline $\mathrm{pH}$ & DPPC/TEMPO & DPPC/Chol/TEMPO \\
& $T_{\mathrm{C}}(\mathrm{K})$ & $T_{\mathrm{C}}(\mathrm{K})$ \\
\hline 1.9 & 316.1 & 319.9 \\
5.0 & 314.0 & 318.0 \\
7.4 & 312.0 & 318.0 \\
8.0 & 311.9 & 316.0 \\
8.4 & 313.9 & 317.9 \\
\hline
\end{tabular}

of partition coefficient $f$, calculated on the basis of EPR spectra, were used to determine the temperature of the phase transition $T_{\mathrm{C}}$ (Table 1).

For the majority of the studied liposomes the decrease of $T_{\mathrm{C}}$ value with increase of $\mathrm{pH}$ of the buffer used for liposome membrane preparation from 1.9 to 8.0 was observed.

\section{Conclusions}

The physicochemical properties of liposomes obtained by mREV method have been determined by the use of the electron paramagnetic resonance (EPR) spectroscopy.

Two spin markers TEMPO and 5-DOXYL have been used. Their properties are similar to those of hydrophilic and hydrophobic drugs. 
The EPR spectra of (5-DOXYL) incorporated into the liposome membranes exhibit anisotropic motion and allowed for the determination of parameter $a_{\mathrm{N}}^{\prime}$, and of the fluidity of the liposome lipid bilayers. The increase of $a_{\mathrm{N}}^{\prime}$ value reflects the rise of polarity of the spin label environment. The EPR spectroscopy showed that fluidity of both types of liposome membranes prepared with and without cholesterol increases with the increase of temperature and depends on $\mathrm{pH}$ and cholesterol content.

The above study confirms that TEMPO can be used to observe the changes in partition between aqueous and fluid lipid regions. The change in the relative value of $f$ as a function of temperature for DPPC shows that in the presence of water excess, this phospholipid undergoes a transition from a "gel phase" to a lamellar smectic liquid crystalline phase.

\section{References}

[1] M. Chung and Y. Chung, Polymerized ion pair amphiphile that shows remarkable enhancement in encapsulation efficiency and very slow release of fluorescent markers, Coll. Surf. B 24 (2002), 111-121.

[2] A.I. Smirnov and P.D. Morse, Very high frequency electron paramagnetic resonance of 2,2,6,6-tetramethyl-1piperidinyloxy in 1,2-dipalmitoyl-sn-glycero-3-phosphatidylcholine liposomes: partitioning and molecular dynamics, Biophys. J. 68 (1995), 2350-2360.

[3] D.D. Lasic, Liposomes: From Physics to Applications, Elsevier, Amsterdam, 1993.

[4] G. Gregoriadis, Liposome Technology, Vol. I, CRC Press, Boca Raton, FL, 1984.

[5] F. Szoka and D. Papahadjopoulos, Procedure for preparation of liposomes with large internal aqueous space and high capture by reverse-phase evaporation, Proc. Natl. Acad. Sci. USA 75 (1978), 4194-4198.

[6] A. Sułkowska, M. Kłoczko and W.W. Sułkowski, Fluorescence study of binding of adenosine derivatives to phospholipid membranes - effect of serum albumin, J. Mol. Struct. 565/566 (2001), 209-212.

[7] W.W. Sułkowski, D. Pentak, K. Nowak and A. Sułkowska, The influence of temperature, cholesterol content and $\mathrm{pH}$ on liposome stability, J. Mol. Struct. 744-747 (2005), 737-747.

[8] W.W. Sułkowski, D. Pentak, W. Korus and A. Sułkowska, Effect of temperature on liposome structures studied using EPR spectroscopy, Spectrosc. Int. J. 19 (2005), 37-42.

[9] R.C. Macdonald and L.R. Hu, Small-volume extrusion apparatus for preparation of large, unilamellar vesicles, Biochim. Biophys. Acta 1061 (1991), 297-303.

[10] D. Marsh, Polarity and permeation profiles in lipid membranes, Biophysics 98 (2001), 7777-7782.

[11] E.J. Shimshick and H.M. Mcconnell, Lateral phase separation in phospholipid membranes, Biochemistry 12 (1973), 23512360.

[12] S. Belsito and R. Bartucci, Sterically stabilized liposomes of DPPC/DPPE-PEG:2000. A spin label ESR and spectrophotometric study, Biophys. Chem. 75 (1998), 33-43.

[13] St.H. Wu and H.M. Mcconnell, Phase separations in phospholipid membranes, Biochemistry 14 (1975), 847-854.

[14] D. Marsh and A. Watts, Evidence for phase boundary lipid. Permeability of Tempo-choline into dimyristoylphosphatidylcholine vesicles at the phase transition, Biochemistry 15 (1976), 3570-3578. 


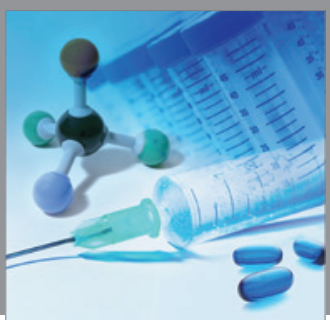

International Journal of

Medicinal Chemistry

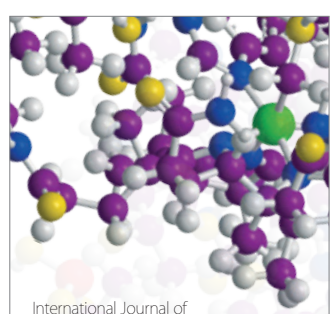

Carbohydrate Chemistry

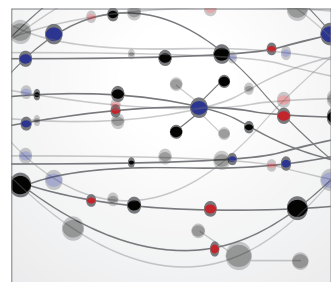

The Scientific World Journal
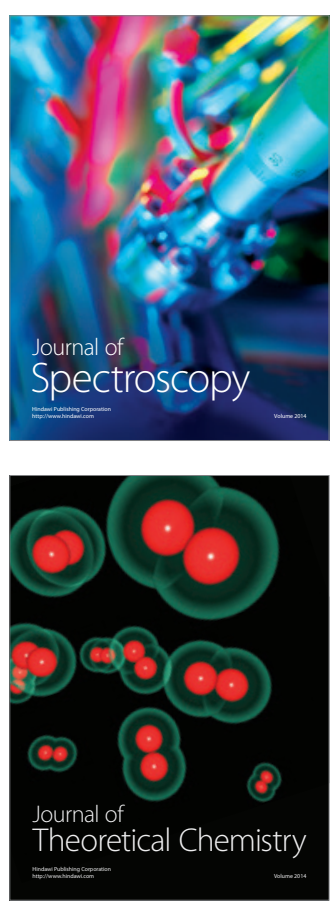
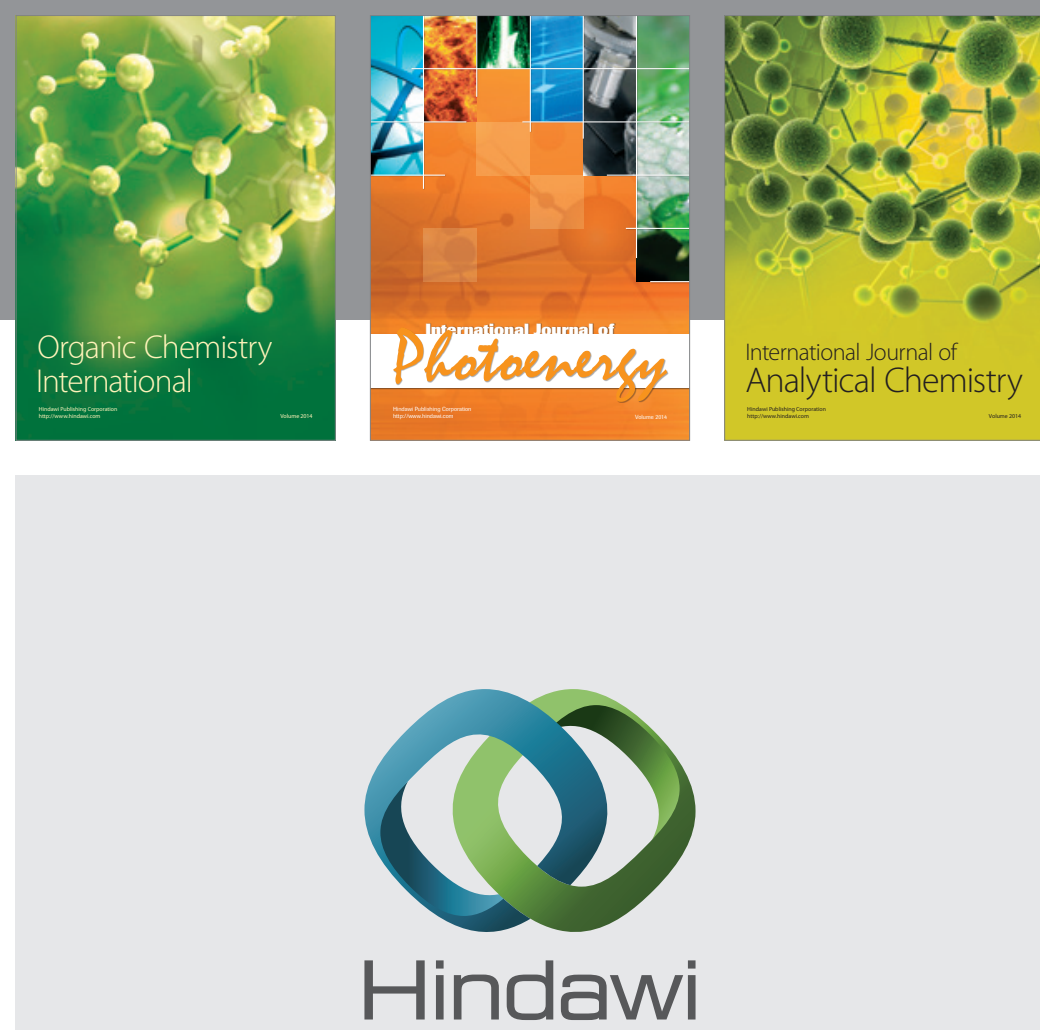

Submit your manuscripts at

http://www.hindawi.com
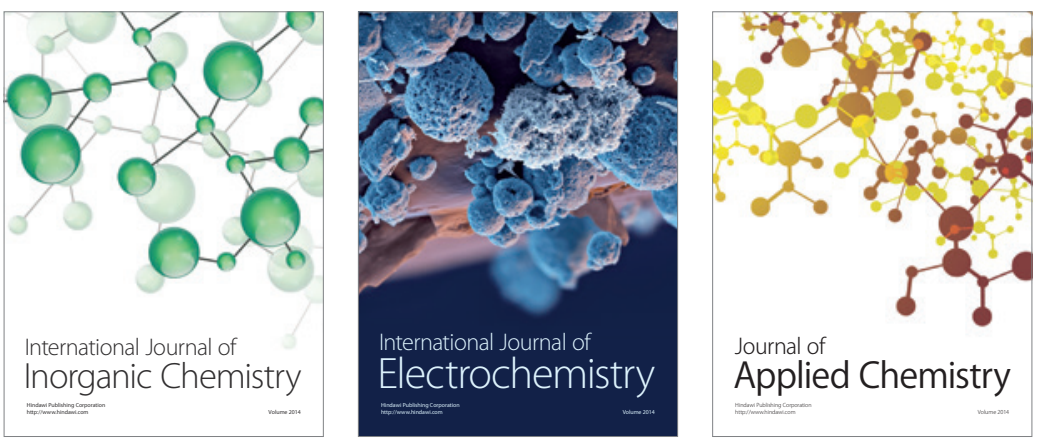

Journal of

Applied Chemistry
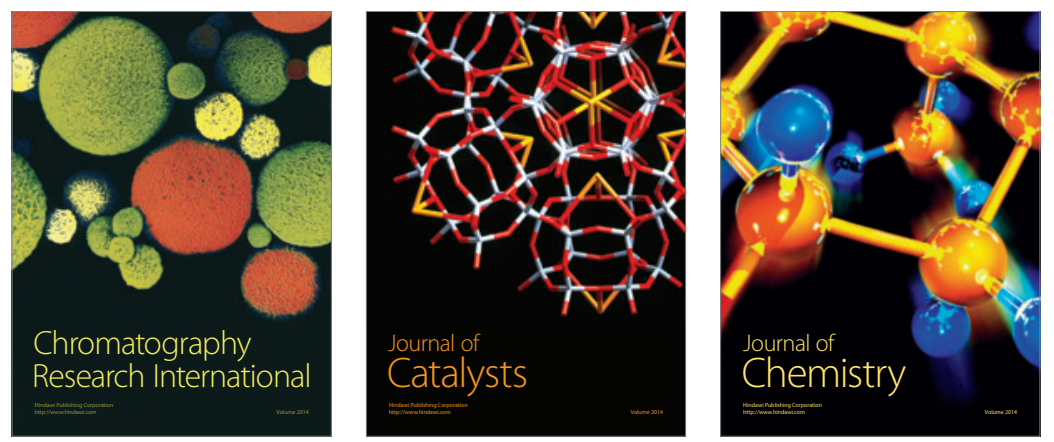
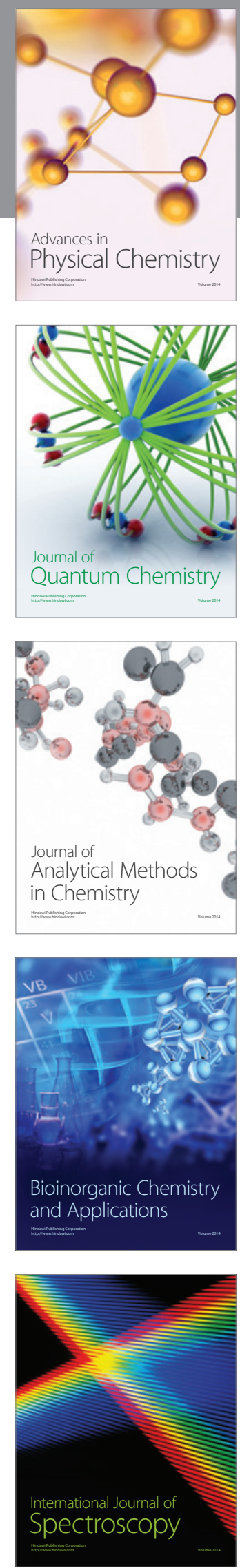